\title{
Erratum
}

J Mol Evol (1983) 19:134-144

\section{Transitions and Transversions in Evolutionary Descent: An Approach to Understanding}

\author{
Richard Holmquist \\ Space Sciences Laboratory, University of California at Berkeley, 1414 Harbour Way South, Richmond, CA 94804, USA
}

Page 134, the first sentence of the Summary should read:

"In this paper I lay a quantitative theoretical groundwork for understanding the proportions of the 12 possible types of base substitutions observed between genes sharing a common ancestor and isolated from extant species."

Page 135, left column, second paragraph, the penultimate sentence should read:

"It is also important for functional regions of the noncoding regions of genes in which that function depends on a sequence of stable base composition."

Page 136, right column, third paragraph, the first sentence should read:
"The explicit form of the function $f$ on the right-hand side of Eq. 1 can be found as follows."

Page 141, Eq. 17:

The middle expression, evaluated at time zero, is equal to $\mathrm{k}$.

Page 141, second line following Eq. 21, in both columns "unknown" should read "unknowns."

Page 141, right column, third paragraph, third line should read:

"...are four independent constraints, a total of 8 independent... ." 